\title{
Looking and thinking when driving: The impact of gaze and cognitive load on steering
}

\author{
Georgios K. Kountouriotis $^{1 *} \quad$ Richard M. Wilkie ${ }^{2} \quad$ Peter H. Gardner ${ }^{2}$ \\ Natasha Merat ${ }^{1}$ \\ ${ }^{1}$ Institute for Transport Studies ${ }^{2}$ School of Psychology \\ University of Leeds \\ ${ }^{*}$ Corresponding author: $g$. kount ouriotiseleeds.ac.uk \\ This article may not exactly replicate the final version published in the Transportation Research Part F: Traffic \\ Psychology \& Behaviour. It is not the copy of record. \\ (c)2015. This manuscript version is made available under the CC-BY-NC-ND 4.0 license \\ http://creativecommons.org/licenses/by-nc-nd/4.0/
}

\begin{abstract}
Driving around bends at high speeds is a task performed by many on a daily basis but the underlying mechanisms of steering control remain largely unknown. Previous research has shown that when steering, gaze direction can be a critical component of success. However, with increased use of in-vehicle information systems (IVIS), there is growing competition over the same resources that are needed to steer (gaze as well as associated attentional resources). Although it can be argued that locomotor steering is an automatic task that can be performed without recourse to conscious 'cognitive' control, much simpler locomotor-related tasks, such as judging one's heading, have been shown to be affected by concurrent attentional tasks (Wann, Swapp, \& Rushton, 2000). Here we examined whether an attentional task placed at an offset fixation point influenced concurrent steering performance along a computer simulated road. The experiments either used gaze-fixation points that had similar properties to real-world road signs (i.e. moved relative to the vehicle) or were more akin to IVIS (i.e. fixed to the vehicle). Results showed that gaze fixation eccentric to future path caused systematic steering biases. The degree or type of cognitive load did not change the degree of steering bias, but there was some evidence of decreased lane variability when viewing the IVIS-type displays. No differences in steering performance were found between the different types of cognitive task. We conclude that where you look is critical for safe driving, and IVIS-type displays might make drivers more susceptible to cognitive interference.

Keywords: Driving; Steering; Locomotion; Gaze; Cognition; Distraction
\end{abstract}

\section{Introduction}

Locomotion, and driving in particular, fundamentally requires the ability to control steering to negotiate bends at high-speeds whilst taking appropriate trajectories along curved roadways. The effect of additional attentional demands imposed by activities such as cell phone use and interactions with invehicle systems have often been studied in terms of reaction times to critical events, such as braking, and lane keeping (e.g., Strayer \& Drews, 2004; Salvucci \& Beltowska, 2008; Recarte \& Nunes, 2003). However, less is known about the effect of cognitive load on steering control, and in particular whether attentional demands interact with the direction of gaze and successful steering control.

Direction of gaze has been shown to be critically important when steering around bends (Land \& Lee, 1994; Wilkie \& Wann, 2003). Although these studies propose two different models of locomotor control, 
both models require the driver to look toward regions of the road ahead. According to the 'Tangent Point' model of steering (Land \& Lee, 1994), drivers should predominantly fixate the apex of the inside road-edge. However, the 'Active Gaze' model of steering (Wilkie \& Wann, 2003; Wilkie, Wann, \& Allison, 2008) proposes a close link between the direction of gaze and the steering trajectory taken by drivers: under conditions of unrestrained gaze, drivers have been found to fixate points on the road that lie on their future path (Wilkie \& Wann, 2003; Wilkie et al., 2008; Wilkie, Kountouriotis, Merat, \& Wann, 2010). The reciprocal also seems to be true: when gaze is forced onto points eccentric to the required curved path, drivers alter their steering trajectories to pass nearer to those fixation points (Kountouriotis, Floyd, Gardner, Merat, \& Wilkie, 2012). This relationship has also been observed when maintaining a trajectory along straight roads (Readinger, Chatziastros, Cunningham, Bülthoff, \& Cutting, 2002). At slow speeds (e.g. when walking) decoupling gaze and steering may have little effect on successful control, however, when driving at high speeds in rapidly changing environments decoupling gaze and steering is not advisable. Of course, in real world scenarios, drivers often have to look away from their future path to perform various tasks, e.g. to sample information from road signs or interact with an in-vehicle satellite navigation system. These tasks require drivers not only to look away from their future path, but to also perform some kind of cognitive task (e.g. read a sign or decipher a map). These concurrent tasks when driving may have an additional effect over and above eccentric fixations, and therefore need to be better understood.

In experimental settings, asking participants to perform a concurrent task whilst driving can result in changes in the natural gaze patterns due to the task itself, e.g. looking at the speedometer. However, even tasks that do not rely on vision and hence do not require participants to direct their gaze towards a specific location (e.g. an auditory in-vehicle information system or talking hands-free on a phone) have been shown to affect gaze behaviours during driving (Victor, Harbluk, \& Engström, 2005). Such tasks can increase the duration of gaze fixations to the road centre, along with a decrease in saccades to the periphery (Recarte $\&$ Nunes, 2003; Victor et al., 2005). Even when gaze patterns do not change, it appears that greater attentional load can reduce the available resources for visual processing of the scene. For example, Strayer, Drews, and Johnston (2003) examined driving performance when drivers talked on a hands-free phone. They observed that participants were less likely to remember seeing billboards in the conditions where they were conversing on the phone compared to the control conditions, even though there was no difference between the two conditions in the number of fixations they made on these objects. Strayer et al. (2003) explain this finding in terms of relocating attention from driving towards the phone task.

In addition to these changes in gaze patterns, cognitive tasks may also change steering, with the variability of lane position reducing under cognitive load conditions (Engström, Johansson, \& Östlund, 2005; Jamson \& Merat, 2005). There is a tight coupling between gaze and steering: not only does steering guide the direction of gaze with drivers looking where they are going (Wilkie \& Wann, 2003, 2005; Wilkie et al., 2010) but gaze direction guides steering with drivers steering where they look (Kountouriotis et al., 2012; Robertshaw \& Wilkie, 2008). A question that arises, therefore, is whether increased cognitive demand affects eye movements (which in turn lead to changes in steering patterns), or whether cognitive demand affects steering patterns (which then leads to changes in eye movements). For example, Engström et al. (2005) used a cognitive load condition which did not require drivers to look at a visual display, but they reported both greater gaze concentration around the road centre and reduced lane keeping variation. Increased gaze concentration has also been shown in other studies using non-visual distraction tasks (e.g., Reimer, 2009; Victor et al., 2005). One possible interpretation of these findings is that the cognitive load increased gaze concentration around the road centre, and this in turn caused steering trajectories to pass closer to the point of gaze (as per Wilkie \& Wann, 2003; Wilkie et al., 2008, 2010).

From this evidence it is difficult to conclude definitively that cognitive load influences steering directly (rather than via gaze mechanisms). Cooper, Medeiros-Ward, and Strayer (2013) examined whether the reduced lateral variation in steering under cognitive load was due to a reduction in lateral eye-movements (gaze concentration). Cooper et al. (2013) instructed drivers to look at an illumi- 
nated number plate on a vehicle ahead. The illuminated plate switched between 3 or 5 vehicles in other lanes and the temporal frequency by which drivers changed their point of gaze from one vehicle to the next was varied. The low frequency gaze changes were supposed to be similar to the patterns exhibited during 'gaze concentration'. This study reported that increased cognitive load did indeed lower steering variability even when gaze changes were matched across 'no cognitive load' and 'high cognitive load' conditions. They concluded that the cognitive task caused attention to be removed from the lane-keeping task, which due to its automaticity improved (Medeiros-Ward, Cooper, \& Strayer, 2014). Whilst it would seem this evidence indicates that the effects of cognitive load are independent of gaze direction, there were some limitations to this study. There were no trials where drivers looked solely towards the road ahead, but in the condition where gaze was predominantly directed to the road ahead ("static predictable") there was no reliable effect of cognitive load. It remains unclear, therefore, whether a cognitive load influences steering when natural gaze behaviours are adopted and drivers fixate their future path. A linked issue is that the non-visual nature of the cognitive task does not generalise to the common situation of retrieving and processing information from the point where you are looking (i.e. there was no task involving information at the point of fixation). Furthermore, Cooper et al. (2013) and Medeiros-Ward et al. (2014) only examined the task of maintaining a trajectory along straight roads. Although this can be a useful paradigm for answering questions related to lane-keeping, the task does not necessarily translate to the more general (and complex) case of steering curved trajectories, where the driver needs to take into account both feedback information from the near road edges as well as prospective information from the road ahead (Land \& Horwood, 1995; Salvucci \& Gray, 2004).

It has to be noted though that whilst a number of studies have shown that a reduction in lane position variability often occurs when cognitive load increases (e.g., Engström et al., 2005; Kubose et al., 2006; Jamson \& Merat, 2005; Merat, Anttila, \& Luoma, 2005), there are also conflicting results-i.e., an increase in lane variability under conditions of cognitive load (e.g., Drews, Pasupathi, \& Strayer, 2008; Salvucci \& Beltowska, 2008). These contradictory findings may be because of differences in task difficulty between various experiments, as well as the specific driving task and scenarios used ${ }^{1}$. Moreover, most of these studies did not specifically examine the task of bend negotiation, which requires continuous online steering control (as opposed to lane keeping in straight roads, where the drivers only need to correct their steering when they have drifted too much towards one side of the lane). Most importantly, however, not all of these studies provide information about where participants were looking during lane keeping, making interpretation of the findings difficult.

The evidence introduced so far suggests that secondary tasks can affect eye-movements either directly by requiring participants to look at an invehicle display (IVIS), indirectly by inducing gaze concentration at the centre of the road, or the secondary tasks can reduce overall the processing of the visual scene. The reason why a secondary task would affect the processing of the scene is not fully understood yet, but one explanation could be the narrowing of the Useful Field of View (UFOV: Ball, Beard, Roenker, Miller, \& Griggs, 1988). The visual area where information can be sampled with one fixation has been named the functional field of view (FFoV), or the useful field of view (Ball et al., 1988). A property of the UFOV is that its size is not constant but instead can decrease in the presence of a cognitive load on the central point of fixation, thus creating artificial tunnel vision (Williams, 1982, 1985). Whilst tunnel vision has been shown in laboratory settings, it is not necessarily the reason for the poor peripheral detection performance during secondary tasks (e.g., Merat \& Jamson, 2008). For example, the 'general interference theory' states that secondary tasks are sometimes allocated more attentional resources and this causes a concomitant decline in the performance of other concurrent detection tasks (Recarte \& Nunes, 2003). In this case, therefore, an increased cognitive load would decrease detection performance in the entire visual field, not just for targets presented in peripheral vision (see Victor, Engström, \& Harbluk, 2008).

The research evidence seems to indicate that in or-

${ }^{1}$ Tasks using different cognitive resources have been postulated to have different effects on steering. Engström et al. (2005) found an increase in lane keeping variation when using a visually demanding task compared to a more cognitive task. However, it should be noted that eye-movements rather than the task itself might have influenced the steering findings. 
der to determine whether cognitive load influences steering, one needs to control gaze direction and eye movements. This can be achieved by enforcing gaze fixation on specific points in the scene, during single task steering, as well as dual task conditions (steering plus cognitive load). When participants are asked to carry out a cognitive task whilst steering, predictions can be made regarding the effect that such a concurrent task will have on steering: (a) indirectly through affecting vision which in turn affects steering performance, and (b) directly, by affecting the control processes underpinning steering itself. In terms of (a) it has been shown that when the boundaries of the road (i.e., the road-edges) are either visually degraded (Kountouriotis et al., 2012) or moved towards peripheral vision (Robertshaw \& Wilkie, 2008), then gaze direction has a greater influence on steering trajectories, with participants exhibiting a greater bias towards offset fixation points. In Kountouriotis et al. (2012) when the road-edges were degraded steering bias towards the fixation points increased (i.e. gaze direction had a greater input into steering than then degraded sources of information. The greater influence of gaze direction has been explained in terms of the Active Gaze model, which combines a number of flexibly weighted perceptual inputs: when one of the inputs (i.e. the road-edges) becomes noisy/unreliable or unavailable, the weighting is increased for other inputs (i.e. gaze direction; Kountouriotis et al., 2012 Wilkie et al., 2008; Wilkie \& Wann, 2002). An increased influence of gaze on steering could also, in theory, be induced without making any physical changes to road-edge luminance or road width, but by asking the drivers to carry out a cognitive or visual task, which would reduce the Useful Field of View (UFOV) and thus degrade the information provided by these features (Figure 4). As such, in a steering task when there is a visual or cognitive load on the point of gaze fixation, if visual information from peripheral vision becomes degraded (i.e., reduced UFOV), then greater steering bias towards the fixation point should be observed.

In terms of scenario (b) - cognitive load affecting steering directly - steering is often viewed as highly automated, without awareness of its procedural aspect and not relying on limited resources (Norman \& Shallice, 2000; Shiffrin \& Schneider, 1977). If this is the case, steering should not be subject to interference from higher-level functions such as cognitive tasks, unless these tasks either affect eye-movements (which have been shown to be crucial for locomotion) or require a motor response (where there would be biomechanical interference with the steering task). There is evidence, however that shows that visualmotor tasks, such as walking (Harley, Wilkie, \& Wann, 2009) and judging one's direction of heading (Wann et al., 2000) can be affected by concurrent cognitive tasks. In the experiments reported here we examined whether the differences in steering patterns observed in the literature when drivers engage in visuospatial tasks and tasks that do not require visual input ('cognitive' tasks) can be attributed to differences in eye-movements as the Active Gaze model of steering predicts. To this purpose, tasks similar to those used by Wann et al. (2000) that affected heading estimation were used, the rationale being that since these tasks can affect a low-level task such as heading perception, they should affect steering too. (Wann et al., 2000) simulated linear self-motion and asked participants to perform one of three concurrent tasks ('Arrow', 'Number' and 'Colour'). They found that two tasks ('Arrow' and 'Number') had a detrimental effect on participants' ability to judge the direction of simulated self-motion, with participants under-estimating the eccentricity of their heading direction. We used similar tasks, as well as an additional auditory task (in Experiment 2) in order to have a condition where attentional focus did not coincide with gaze focus.

In the first experiment reported here visual tasks were placed at fixation points at a constant angle relative to the driver (similar to looking at an IVIS; as used by Readinger et al., 2002) and in the second experiment the fixation points were positioned in relation to the world at a fixed distance from the driver (as in Kountouriotis et al., 2012; Robertshaw $\&$ Wilkie, 2008). The same fixation points were used in baseline conditions (without additional cognitive load) as well as in the cognitive task conditions, allowing the impact of cognitive load and gaze direction on steering to be quantified.

\section{Experiment 1: Fixations relative to the vehicle ('IVIS')}

In the first experiment participants steered a series of bends whilst directing their gaze on to a fixation 
cross displayed in one of four possible eccentricities. The fixation point was always at a constant angle to driver, mimicking the properties of an in-vehicle information system. In the baseline conditions participants were asked to simply steer while fixating their gaze on the fixation point, while in the task conditions they had to perform one of two tasks (judge the direction of arrows or a number series).

\section{Methods}

\section{Participants}

Fourteen volunteers took part in this study, eight of them were females. The mean age was $23.93 \pm 4.86$ years and all had a driving license (one participant held a learner's licence at the time of testing). All of the participants were naïve to the purpose of the experiment.

\section{Apparatus}

A PC with Intel i7 $950(3.07 \mathrm{GHz})$ processor generated images at $60 \mathrm{~Hz}$ with a resolution of $1280 \times 1024$ pixels. Images were created using WorldViz Vizard 3.0. Images were projected using a Sanyo Liquid Crystal Projector (PLC-XU58) onto a back projection screen with dimensions of $1.98 \times 1.43 \mathrm{~m}$. Participants sat $1 \mathrm{~m}$ away from this screen, making the total field of view $89.42^{\circ} \times 71.31^{\circ}$. A height-adjustable racing-style driving seat was used to maintain eyeheight $1.20 \mathrm{~m}$ above the ground. Participants sat in a matt black booth so that the projection provided the only source of light. Steering was controlled using a force-feedback wheel (Logitech G27). Gaze data were recorded using a remote Applied Science Laboratories 504 gaze monitoring system, which uses pan-tilt tracking to follow eye movements and was calibrated on a 9-point grid.

\section{Visual Stimuli}

The environment consisted of a $3 \mathrm{~m}$ wide bending roadway and participants were instructed to steer along the centre of the road. The road consisted of a $9 \mathrm{~m}$ long straight section and after that a single bend. The road curvature started at $60 \mathrm{~m}$ but it reduced at a constant rate of change of $-25 / \pi \mathrm{m}$. This type of bend was used over bends with a constant curvature, where (in theory) participants can steer by keeping the steering wheel at a constant angle. This should make the steering task more demanding by requiring participants to constantly monitor that the turning rate is sufficient. Figure 1 shows a top-down view of the bends used here, compared to a bend of constant curvature for comparison. In half the trials bends were flipped across the long axis to make bends curve to the right or the left. The speed was kept constant in all of the conditions at $13.8 \mathrm{~m} / \mathrm{s}$ to avoid participants negating the difficulty of the steering task by slowing down in the cognitive task conditions. Each trial lasted seven seconds.

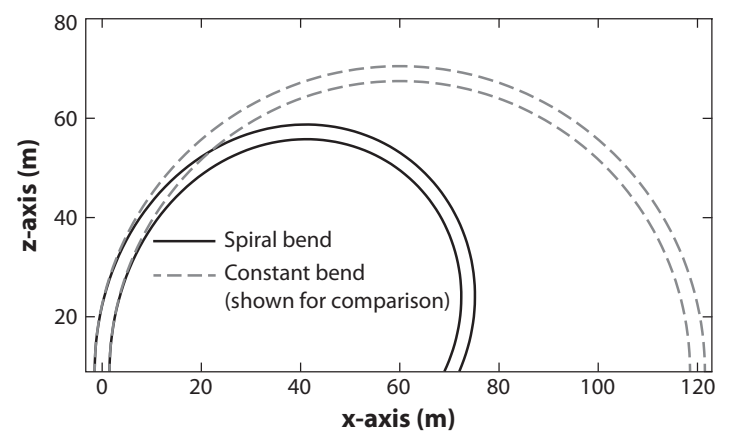

Figure 1 Example of a spiral bend (where the radius reduces at a constant rate) compared to a bend of constant curvature.

The fixations used in this experiment were at a constant angle from the driver irrespective of the scene in order to imitate the properties of in-vehicle information systems, such as the position of a satellite navigation system on a windscreen.

Four different eccentricities were used, set at $20^{\circ}$ or $30^{\circ}$ from the driver's midline, either at the same direction of the bend (named Inside and Far Inside respectively), or opposite to the direction of the bend (named Outside and Far Outside respectively). For example, in a right bend, the fixation point located at $20^{\circ}$ to the right of the participant would be the Inside fixation whereas the fixation pointed located $20^{\circ}$ to the left of the participant would be the Outside fixation. Figure 2 shows the scene with all four fixation points (only one fixation was visible during each trial).

Fixations with a constant offset angle from the driver were also used by Readinger et al. (2002). In their study, participants steered towards the fixation points when driving along a straight road, with the 


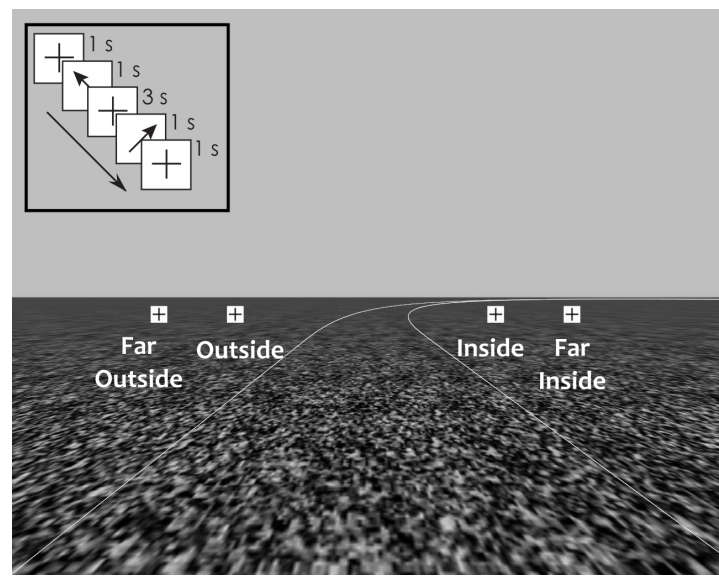

Figure 2 The four possible fixation points in Experiment 1 were always at the same offset angle ( 20 or 30 degrees in either direction) relative to the driver's midline. Only one fixation was visible during each trial. The insert shows an example sequence for the 'Arrow' condition, where the correct response (after locomotion finishes) would be 'clockwise' (right button press). The same timings were used in all task conditions.

magnitude of bias generally being in proportion to the magnitude of the offset of the fixation point. However, bias reached a plateau on the more extreme offset fixations (not used here). If similar results are observed in this experiment, then systematic negative biases for Far Outside and Outside fixations are expected, and positive biases for the Inside and Far Inside fixations. Furthermore, the magnitude of these biases should reflect the offset of the fixations larger (positive) values for the Far Inside compared to the Inside fixation, and larger (absolute) values for the Far Outside compared to the Outside fixations.

\section{Cognitive Tasks}

Two cognitive tasks were used in this experiment, 'Arrow' and 'Number', as well as a control condition, during which the participants were asked to keep their gaze fixating the target throughout the trial. The cognitive task conditions presented information on the fixation target which were similar to the Arrow and Number conditions used by Wann et al. (2000). Here, however, instead of asking the participant to report the next arrow or number in a series, as in Wann et al. (2000), the participants were asked to compare either two arrows (Arrow condition) or two numbers (Number condition) presented at different times during the trial and make a judgement about the two stimuli. The onset of the first stimulus was two seconds into the trial, and the onset of the second stimulus was six seconds into the trial. Each stimulus was visible for one second. When no arrow or number was present the fixation point displayed a cross. At the end of the trial participants reported whether the numbers ascended or descended, or whether the arrows were moving clockwise or anticlockwise. The responses were made by pressing the right paddle of the steering wheel for ascending or clockwise and the left paddle for descending and anticlockwise. Participants were allowed to practice the tasks until they felt comfortable performing them whilst steering. The insert of Figure 2 shows the timings of an example Arrow condition.

Since participants had to wait until the end of the trial to make a response, reaction times do not act as a useful measure in these experiments. In order to get an estimate of the relative difficulty of each task we measured separately reaction times and accuracy rates to respond purely to the visual stimuli (using the same display timings as in the actual experiment but without a concurrent steering task). Accuracy was similar across tasks, though RTs were quicker for 'Number' trials and slowest for 'Arrow' trials. The full results of this pilot are reported in the methods section of Experiment 2.

\section{Experimental Design and Analysis}

A blocked design was used to separate the task and no task (baseline) conditions, in order to avoid situations where in a 'No Task' condition participants expect a stimulus to appear on the fixation point and therefore allocate more attentional resources than they would do otherwise. This provides us with a relatively pure measure of steering when fixating the signs without additional cognitive load. To minimise the confounding effects of practice and fatigue the no-load condition was repeated twice, at both the beginning and end of the testing session. Participants completed the 'No Task 1' block with the control conditions ( 6 trials of 4 fixations, half in right bends and half in left bends). Then in the second block ('Task' block) they completed the two cognitive tasks randomly interleaved ( 6 trials of 4 fixations $\times 3$ tasks, half of them right bends and half left). Finally, the 
'No Task 2' block repeated the control condition (6 trials of 4 fixations).

\section{Results and Discussion}

\section{Cognitive Task Performance}

In order to examine performance during the two cognitive tasks, accuracy scores (i.e., the percentage of correct responses) were calculated. The proportion of correct responses averaged across participants for the Arrow and Number tasks were $91.07 \%$ and $93.15 \%$ respectively, indicating that the participants performed well in both tasks, and to a similar level as when performing the tasks without steering (see Table 1, Experiment 2 for a pilot experiment examining all secondary tasks without steering). A paired-samples $t$-test found no significant difference between the Arrow and Number tasks, $t(13)=-1.71, p=.110$.

\section{Steering Bias}

Steering bias is a directional measure of steering error, measuring the overall tendency of the driver to steer towards the outside road edge (negative values), or the inside road edge (positive values) relative to the centre of the road (zero bias). A 4 (levels of Task: No Task 1, Arrow, Number and No Task 2) by 4 (levels of fixations: Far Inside, Inside, Outside and Far Outside) repeated-measures ANOVA was carried out on the steering bias values. There was no significant effect of Task, $F(3,39)=1.92, p=.143$, but a significant main effect of Fixation, $F(3,39)=3.55, p=$ $.023, \eta_{p}^{2}=.21$, and a significant interaction between Task and Fixation, $F(9,117)=2.41, p=.015, \eta_{p}^{2}=.16$, shown in Figure 3.

When No Task 1 is removed from the ANOVA so that Task has only three levels and Fixation four levels, the interaction between Fixation and Task ceases to be significant, $F(6,78)=1.04, p=.405$. It seems then that the interaction is driven by the behaviour of the participants in the No Task 1 condition/block where there are systematic directional steering biases caused by fixation direction (white triangles on Figure 3). Fixating Far Outside causes the greatest understeer (i.e., steering towards the outside edge) and fixating Far Inside causes the greatest oversteer (steering towards the inside edge) with the intermediate conditions causing smaller biases in the

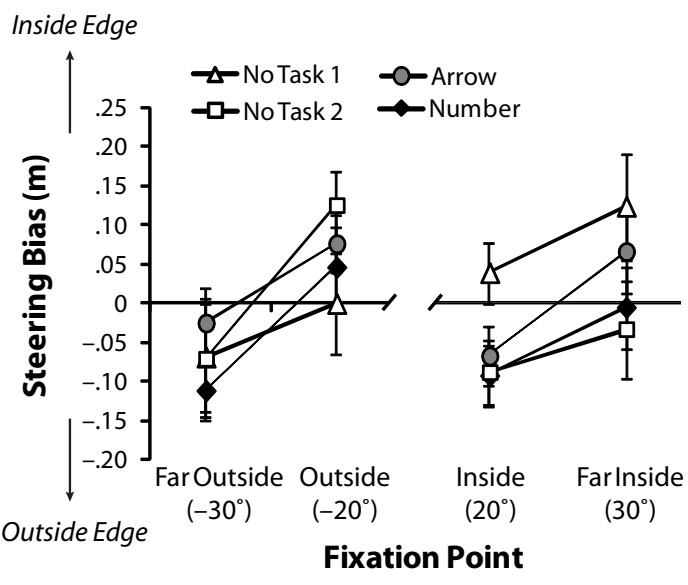

Figure 3 The interaction between Fixation and Task conditions in steering bias. When participants fixate on the Inside they steer towards the inside road edge in No Task 1 relatively to the rest of the task conditions, and they also understeer in the Outside fixation condition compared to the rest of the task conditions. Error bars $=S E M$.

direction of fixation. This is the result that would be expected if participants steered in the direction of gaze (Readinger et al., 2002; Robertshaw \& Wilkie, 2008; Kountouriotis et al., 2012).

For all other conditions (Tasks and No Task 2) participants did exhibit some degree of bias caused by fixation direction. This is shown by the direction of slopes between Far Inside and Inside fixation (Figure 3, greater oversteer in Far Inside), and Outside and Far Outside (greater understeer in Far Outside than Outside). However, there is a second factor influencing steering that causes participants to steer away from the direction of gaze - this is most obvious in the Outside and Inside fixation conditions comparing No Task 2 with No Task 1 (Figure 3, white squares vs. white triangles).

We believe that this steering bias is due to a property of the static fixation points used. If the driver steers in the opposite direction of fixation then this causes a change in the scene and makes the centre of the road lie closer to the point of gaze. This means that the road will fall closer to central vision and so may provide more useful information to participants (Figure 4 illustrates this effect). The fact that this behaviour is not exhibited in the No Task 1 block (which was always presented first) indicates that steering away from gaze may be a learnt response to try to 
reduce the biases caused by eccentric fixation. It appears therefore that participants learnt to alter their natural steering behaviour in order to 'move' the road towards their point of gaze. This demonstrates remarkable flexibility in the coordination of gaze and steering.

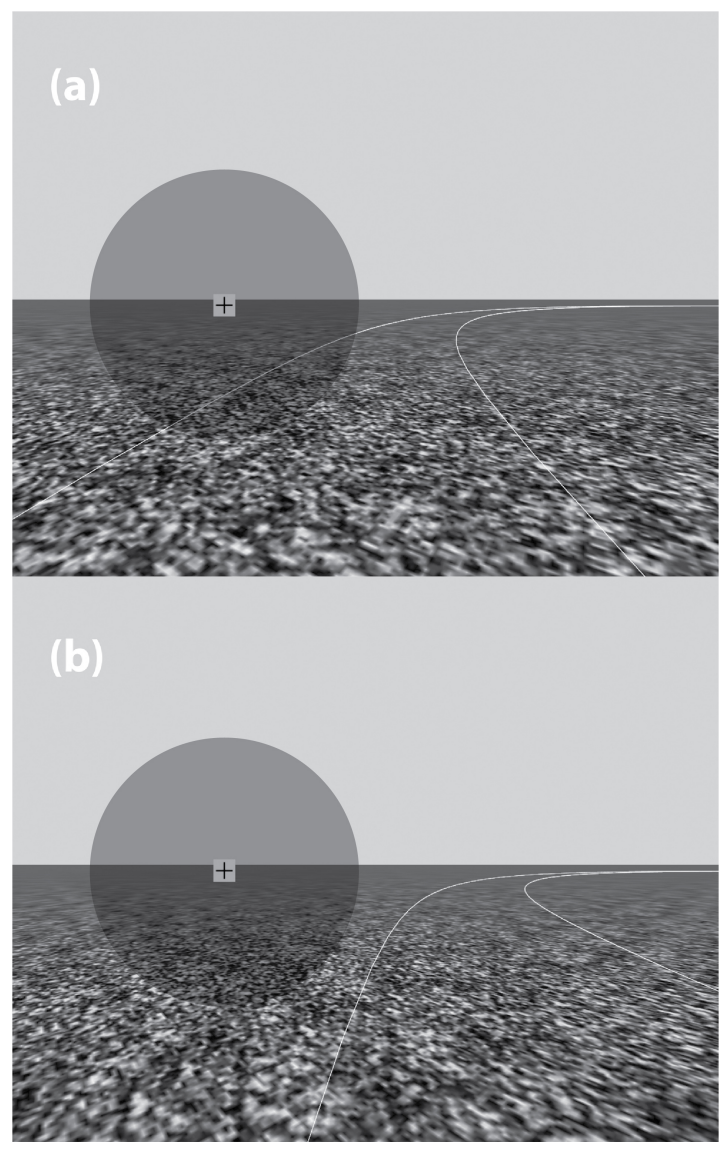

Figure 4 Over/under-steering and the relation of the fixation point to the road. (a) Steering away from the point of gaze means that the road is closer to central vision (see shaded circle). (b) Steering towards the point of gaze moves the road towards peripheral vision where not only there is less visual acuity but the gaze angle hasn't been reduced. The dark grey discs demonstrate a $\sim 30^{\circ}$ zone indicating central vision (these discs were not visible to the partici pants).

It was expected that due to a narrowing of the UFOV (Ball et al., 1988; Williams, 1982, 1985) participants' steering would be influenced more by gaze direction (with participants exhibiting greater biases towards the fixation points) when a concurrent task was present compared to the No Task conditions. There were, however, no differential effects of cognitive task on steering bias.

\section{RMS Steering Error}

RMS error is calculated by taking the square of the signed steering error (bias) for every frame, averaging across frames and then taking the square root. It provides an overall estimate of how close the participant was to the centre of the road in a given trial but is unsigned (in contrast to bias which shows whether there was systematic over/understeer but underestimate large errors that are not biased). Low RMS scores indicate participants stayed near the centre of the road whereas high values indicate participants were away from the centre. As with steering bias, a 4 (levels of Task) by 4 (Fixations) repeatedmeasures ANOVA was carried out on the RMS values. There was a significant main effect of Task conditions, $F(3,39)=14.10, p<.001, \eta_{p}^{2}=.52$, as well as a significant main effect of Fixation conditions, $F(3,39)=8.31, p<.001, \eta_{p}^{2}=.39$. No significant interaction was found between these two factors, $F(4.23,55.01)=0.47, p=.764$.

The main effect of Fixation is shown in Figure $5 \mathrm{a}$. Repeated planned contrasts indicate that RMS error in the Inside fixation condition was significantly lower both from the Far Inside fixation, $F(1,13)=$ $23.41, p<.001$, and the Outside fixation condition, $F(1,13)=10.94, p=.006$. No difference was found between the Outside and Far Outside fixations, $F(1,13)=1.96, p=.185$. The lower RMS error in the Inside fixation condition is in line with the findings of Kountouriotis et al. (2012), where fixation on the inside of the bend produced lower RMS scores than fixation on the outside of the bend. It appears, however, that the advantage of fixating on the inside of the bend is lost when the fixation is moved further away from the road centre (Far Inside fixation).

The main effect of Task on RMS error is shown in Figure 5b. We first compared the two task conditions using a $t$-test which showed no difference between the two, $t(13)=.62, p=.55$. Therefore we collapsed these two conditions and ran a 3-way ANOVA comparing No Task 1, Tasks, and No Task 2 which was significant, $F(2,26)=12.66, p<.001, \eta_{p}^{2}=.49$. Pairwise comparisons with Sidak correction showed that No Task 1 was significantly higher than Tasks 


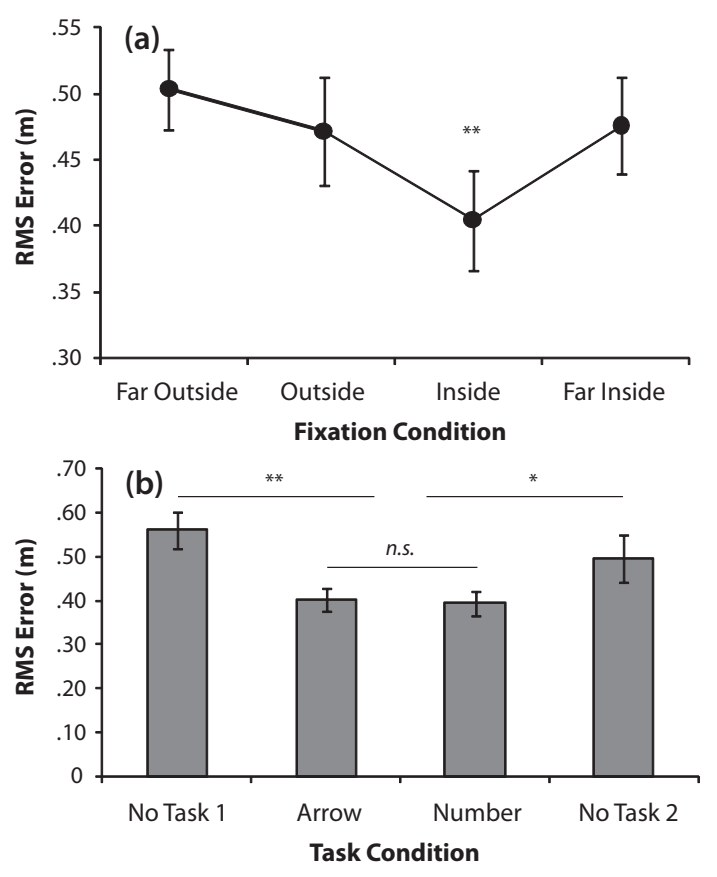

Figure 5 RMS steering error. (a) The main effect of Fixation. Participants perform better when they fixate on the inside of the bend, but this advantage of the inside fixation does not exist on the more extreme Far Inside fixation. (b) The main effect of Task. Participants' RMS errors are lower when they perform a secondary task (either Arrow or Number) compared to the conditions where there is no concurrent task. Error bars = SEM.

$(p<.001)$, No Task 2 was significantly higher than Tasks $(p=.034)$ but there were no differences between No Task 1 and No Task $2(p=.276)$.

An interesting phenomenon occurs here: RMS scores, usually indicative of steering error and variability in steering performance, are higher when participants do not perform a secondary task. As discussed in the Introduction, this finding can be explained by previous research. One measure that might help interpret the present findings is steering reversal rate (McLean \& Hoffmann, 1975).

\section{Steering Reversals}

If RMS error reflects reduced steering variability in the tasks conditions compared to baseline then we should see the same pattern when using a measure such as steering reversals. Steering reversal rate can be defined as the number of times per minute that the direction of the steering wheel changes by a finite amount - from half a degree to 10 degrees (McLean \& Hoffmann, 1975; Macdonald \& Hoffmann, 1980). In our experiment trials only lasted for a relatively short duration (7s), so we generated a measure of steering reversals using the rate of change of the steering wheel angle and then identifying any peaks above $1^{\circ}$ in the opposite direction of the required steering response. We used these criteria in order to capture instances where participants needed to make major adjustments to their trajectory rather than capturing overall steering input (though bidirectional reversals of $0.5^{\circ}$ and $0.1^{\circ}$ revealed similar patterns as in the reported metric). Similar to the analysis of RMS steering error, a $t$-test was carried out to compare the difference in number of steering reversals (see Figure 6) between Number and Arrow, which showed no significant difference, $t(13)=-.56, p=.588$. Therefore we collapsed across the tasks conditions and ran a repeated-measures ANOVA with one factor with 3 levels (No Task 1, Tasks, No Task 2). There was a significant main effect, $F(2,16)=17.61, p<.001, \eta_{p}^{2}=.575$. Pairwise comparisons with Sidak corrections showed that differences were significant between all three conditions. Participants in the No Task 1 condition made more steering reversals compared to the Tasks condition $(p<.001)$, and also more steering reversals compared to No Task $2(p=.027)$. Additionally, in No Task 2 participants made more steering reversals compared to the Tasks $(p=.032)$.

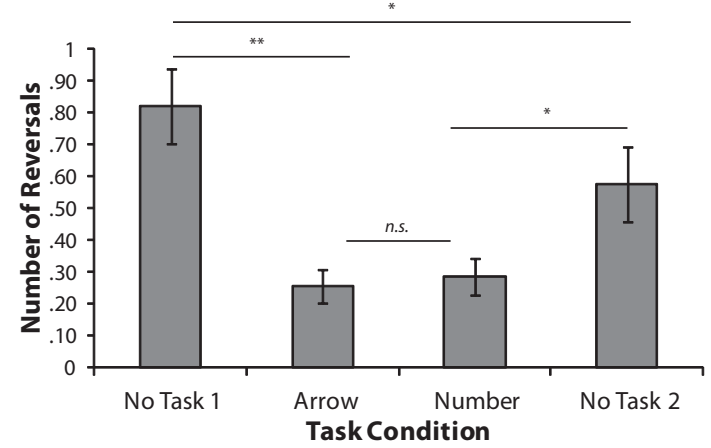

Figure 6 Number of steering wheel reversals for each Task condition. Participants perform fewer steering reversals when there is a secondary task compared to the conditions without a concurrent task. Error bars $=S E M$. 
When participants had a cognitive load they made fewer steering reversals compared to the conditions without a cognitive load. This finding is in line with the observations of Macdonald and Hoffmann (1980) who suggested that increasing the demands on a steering task with a cognitive load causes a reduction in steering reversals; they argue that this happens because when participants have to perform a demanding concurrent task they remove their attention from the steering task and therefore make fewer corrective adjustments. The alternative explanation is that steering performance is actually improving when attention is shifted away, because steering is such a highly-automated task (Medeiros-Ward et al., 2014). This argument equates reduced variability with better performance, but this relationship is commonly observed in experts performing a number of skilled visual-motor tasks, for example baseball (Gray, 2004), golf and football (Beilock, Carr, MacMahon, \& Starkes, 2002) as well as typing (Tapp \& Logan, 2011).

\section{Experiment 2: Fixations in rela- tion to the world ('Road Signs')}

The first experiment showed that fixations on signs at a constant angle relative to the observer led to reduced steering corrections in the task conditions. In terms of directional errors (steering bias), participants steered in the direction of gaze in the first experimental block, but in the following blocks they steered in a way that would bring relevant information closer to central vision. In the second experiment the fixation points were positioned in the world, and their position updated every frame so they were always at a fixed distance ahead of the observer. Additionally, being positioned in the world they created a gaze angle that could be reduced if the participant steered towards them (see Kountouriotis et al., 2012; Robertshaw \& Wilkie, 2008). This means that participants could close down the angle between their mid-line and gaze direction. These fixation points have similar properties to road signs, in that drivers can reduce their gaze angle by steering towards them (although unlike real signs these fixation points were always kept a set distance away and were always perpendicular to the observer to ensure clear and consistent visibility of the sign).
In Experiment 2 we also decided to employ an additional task: participants looked at the same fixation points, but were asked to evaluate auditory information rather than visual information ('Sound' task). The form of the task was similar to the visual task: participants heard two sounds of different frequencies and after trials finished they had to indicate whether the second sound had a lower or higher frequency than the first (using left or right button presses). The rationale for using this task was that the Number and Arrow tasks should draw attention to the fixation point, but the Sound task should not. Therefore, according to the UFOV theory, no narrowing of the visual field should be observed in the Sound task, which would make participants less likely to exhibit large steering biases towards the point of fixation.

In order to accommodate fixations located at the centre of the road, the road width was increased by $1 \mathrm{~m}$ (4 m wide, compared to $3 \mathrm{~m}$ wide in Experiment 1). This increase in road width should allow for higher RMS/steering variability errors, which could differentiate between the different cognitive tasks (something Experiment 1 did not show and could have been due to the relatively narrow roads used).

\section{Methods}

\section{Participants}

Twelve participants took part in this study (six females) with a mean age of $20.5 \pm 0.79$ years. Ten participants held a valid driving license at the time of testing. The two non-drivers did not perform differently from the drivers ${ }^{2}$. All of the participants were naive to the purpose of the experiment.

\section{Apparatus}

The same apparatus was used as in Experiment 1.

\section{Visual Stimuli}

The same bends as Experiment 1 were used, with the only difference being that the roads were $4 \mathrm{~m}$ wide (instead of $3 \mathrm{~m}$ wide in Experiment 1) in order to accommodate for the different fixation points used here.

${ }^{2}$ We re-ran all analyses excluding the participants without a driving licence, but this had no effect on the pattern of the statistical results. 
The fixations used in this experiment had different properties compared to the fixations used in the previous experiment. Three fixation points were used, one in the centre of the road approximately $16.1 \mathrm{~m}$ ahead of the participant, and two either side of the road, $2 \mathrm{~m}$ outside either road edge. These fixation points were not fixed on the ground, instead their position was updated on every frame so they maintained the same distance in front of the driver. How ever, they were also linked to the world so if the driver steered towards the fixation point the angle of gaze would reduce.

\section{Cognitive Tasks}

Three cognitive tasks were used in this experiment, the Arrow and Number tasks from Experiment 1, and an additional auditory task (Sound task). This task was added in order to examine whether steering control is affected by a cognitive task that does not require any visual processing. A simple fixation cross was used to control the location of participants' gaze throughout the Sound task, similar to the other tasks. Participants heard two tones presented sequentially at different frequencies (eight tones were used ranging from $240 \mathrm{~Hz}$ to $590 \mathrm{~Hz}$ in $50 \mathrm{~Hz}$ increments). At the end of the trial participants indicated if the second sound had a higher or lower frequency than the first. Participants responded in the same way as in the Number task by pressing the right paddle for 'higher' and the left paddle for 'lower'. The timings of the Sound task were identical to the Arrow and Number tasks (see Figure 2) with each sound lasting for $1 \mathrm{~s}$.

As with Experiment 1, a blocked design was used. First, participants completed the control conditions ( 6 trials of 3 fixations) (No Task 1 block). Then in the second block the three cognitive tasks were randomly interleaved ( 6 trials of 3 fixations $\times 3$ tasks). Finally, they repeated a control block with no cognitive task (6 trials of 3 fixations) (No Task 2). Conditions within each block were always randomised and half of the 6 trials of every condition were right bends and half of them left bends.

Since participants had to wait until the end of each trial to respond to the tasks, it is not useful to measure reaction times for these tasks. Therefore a pilot study was conducted using these tasks (with the same timings as in the actual experiments) but without any steering. Ten participants (mean age $=21.6$ years) sat in the driving simulator and were presented with eight trials of each of the three cognitive tasks. Participants were asked to respond as quickly and accurately as possible at the onset of the second stimulus. The reaction times and percent correct results are shown in Table 1 . Two repeated-measures ANOVAs were conducted, one for reaction times and one for accuracy. A significant main effect was found on reaction times, $F(2,18)=4.99, p=.019, \eta_{p}^{2}=.36$, but no significant differences were found on the accuracy scores, $F(2,18)=1.56, p=.237$. Pairwise comparisons with LSD adjustment showed that participants were significantly slower in the Arrow task compared to the Number task $(p=.026)$; no other differences were found. The results indicate that although participants took more time to make a judgement on the relative directions of the arrows in the Arrow task, the three tasks gave similar results in terms of accuracy.

Table 1 Mean and Standard Deviation of Reaction Times and Accuracy scores for the three cognitive tasks without steering.

\begin{tabular}{lccccc}
\hline & \multicolumn{2}{c}{ Reaction Times (s) } & & \multicolumn{2}{c}{ Accuracy (\%) } \\
& $\bar{x}$ & $S D$ & & $\bar{x}$ & $S D$ \\
\cline { 2 - 3 } \cline { 5 - 6 } Number & 0.69 & 0.14 & & 94.17 & 10.43 \\
Arrow & 1.01 & 0.47 & & 90.00 & 8.61 \\
Sound & 0.82 & 0.30 & & 95.00 & 9.96 \\
\hline
\end{tabular}

\section{Results and Discussion}

\section{Cognitive Task Performance}

Performance in the three cognitive tasks during the steering study was assessed using accuracy scores (i.e., the percentage of correct responses). The accuracy scores were analysed using a repeated-measures ANOVA with three levels. No significant difference was found between tasks in terms of accuracy, $F(2,22)=0.68, p=.516$, consistent with the results obtained without steering (see Table 1). Although it appears that accuracy scores were lower in Experiment 2 compared to the results with no steering (Number: mean $=80.09 \%, S D=26.95$; Arrow: mean $=73.61 \%$, SD $=26.50$; Sound: mean $=75.46 \%$, SD 
$=25.34)$ this was largely driven by four participants making incorrect response in approximately half of the trials. We re-ran all of the following analyses without these participants and the patterns remained largely unaffected.

\section{Steering Bias}

A 3 (fixation points: Inside, Centre, Outside) by 5 (task conditions: No Task 1, Arrow, Sound, Number, No Task 2) repeated-measures ANOVA was conducted on the steering bias values. There was a significant main effect of Fixation, $F(2,22)=11.29, p<$ $.001, \eta_{p}^{2}=.51$, with participants steering towards their point of gaze (see Figure 7a). There was also a main effect of Task, $F(4,44)=3.10, p=.025, \eta_{p}^{2}=$ .22 , shown in Figure $7 \mathrm{~b}$ with a general pattern of oversteer (i.e., steering towards the inside road edge). There was no significant interaction between Fixation and Task, $F(8,88)=.81, p=.593$.

The main effect of Fixation on steering bias was examined using planned contrasts. A significant difference was found between the Outside and Central fixations, $F(1,11)=15.54, p=.002$, but no significant difference was found between the Central and Inside fixations, $F(1,11)=0.78, p=.397$. This pattern is similar to the steering behaviour exhibited in Kountouriotis et al. (2012), and can be explained by the tendency of drivers to oversteer when negotiating bends (as observed in Wilkie et al., 2010; Raw, Kountouriotis, Mon-Williams, \& Wilkie, 2012).

The significant main effect of Task (Figure 7b) was analysed using pairwise comparisons with Sidak correction. The only significant difference was between the No Task 1 and Sound conditions, $p=$ .047. Across all fixation conditions, participants oversteered more in the Sound condition compared to the No Task 1 condition where they stay closer to the centre of the road.

The fact that no significant interaction was found between Task and Fixation conditions indicates that gaze had a similar effect across the Task conditions. This does not match the predictions of attention load models of cognition. There appears to be no 'tunnelling effect' (reduced UFOV) in this experiment. However, in order to draw any firm conclusions on the effect of the cognitive tasks on steering these results have to be examined along with other steering metrics, namely RMS steering error and steering reversals.

\section{RMS Steering Error}

A 3 (fixation points) by 5 (task conditions) repeatedmeasures ANOVA was conducted for the RMS steering error. There was no significant main effect of Fixation, $F(2,22)=2.52, p=.103$, no significant main effect of Task, $F(2.22,24.43)=0.926, p=.458$, and no significant interaction between Fixation and Task conditions, $F(8,88)=1.73, p=.103$.

These findings are in contrast to Experiment 1, where in the Arrow and Number conditions participants had lower RMS errors compared to the No Task conditions. The differences between the two experiments were the inclusion of an auditory task in Experiment 2 , the wider road, and the different properties of the fixation points. There is no reason to assume that including a third task would lower participants' RMS errors in the other two tasks. The wider road used should in theory increase RMS errors overall (because road-edges are moved further into peripheral vision, see Robertshaw \& Wilkie, 2008; Kountouriotis et al., 2012), but also it should allow for more variation in RMS errors and thus accentuate any differences between task conditions that could have been missed in narrower roads. Instead, we believe that reduced errors may be due to the nature of gaze fixation in this task. The fixations used in this experiment biased steering trajectories towards the direction of participants' gaze, and that reduced the gaze angle offset. When the fixations are set at a constant offset angle from the participants (like in Experiment 1), the gaze angle is never reduced. If participants steer in the direction of gaze the offset gaze angle does not close down, and the more they steer towards the fixation point, the more this trajectory moves away from the centre of the road. Therefore, drivers seemed to develop a strategy (described in Experiment 1) to mitigate the adverse effects of steering indefinitely towards their fixation point. This strategy is potentially the reason why there is a difference in RMS steering error between the Task conditions in Experiment 1.

\section{Steering Reversals}

Given the strong link between RMS steering error and steering wheel reversals observed in Experiment 1 , no differences are expected in the number of 

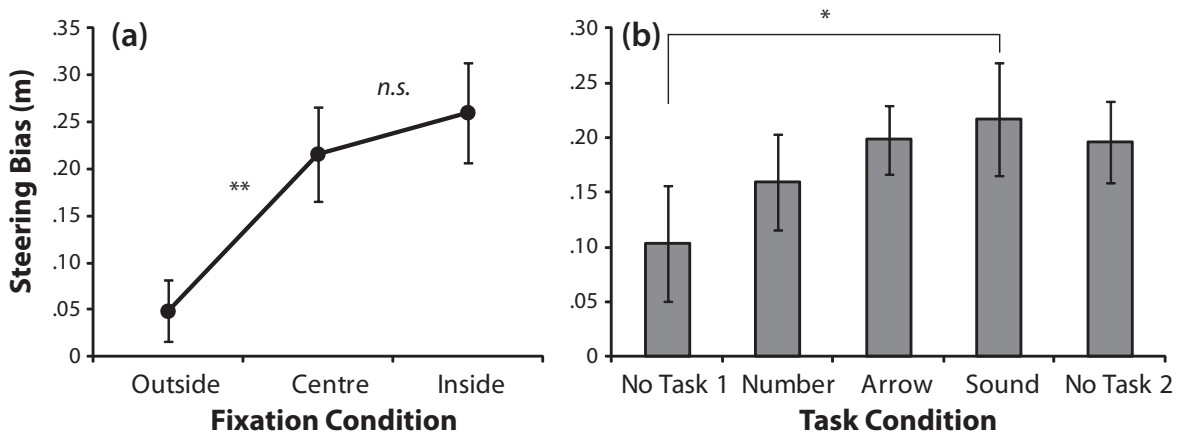

Figure 7 Steering Bias results for Experiment 2. Positive values indicate oversteer trajectories that cut closer to the inside of the bend than the middle of the road. (a) The main effect of fixation collapsed across Task conditions, whereby participants steer more towards the inside of the bend, when the fixation point is positioned beyond the inside edge of the road. (b) The main effect of Task collapsed across fixation conditions. Participants oversteer more in the Sound task compared to No Task 1 . Error bars = SEM.

reversals in the different task conditions, similar to the non-significant findings in RMS steering error in this experiment. Indeed, the 5-level (No Task 1, Arrow, Number, Sound, No Task 2) repeated-measures ANOVA used to analyse the steering reversals in this experiment showed no significant effect of Task, $F(1.90,20.99)=0.54, p=.707$. In fact, participants made almost no steering reversals as defined in the previous experiment: the average reversals (across 3 fixations, 6 trials each) for No Task 1 was 0.02 , for Number 0.03, for Arrow 0.03, for Sound 0.04, and for No Task 2 0.02. In Experiment 1, the lowest number of reversals was 0.30 in the Arrow condition (see Figure 6).This lack of steering reversals is probably due to the different fixation points used here, as discussed above.

By using offset fixations where the driver could reduce the offset of the gaze angle - by steering closer to the fixation point - the steering task seems to have been easier compared to Experiment 1 where the offset gaze angle could never be reduced. This could be the reason for the lack of an effect of concurrent tasks in Experiment 2. These results indicate that looking on a point in the world (and not on an in-vehicle information system) may result in better steering.

\section{General Discussion}

Our research examined the effects of a number of cognitive tasks on steering. Unlike previous work, we recorded steering along a set of bending roadways when gaze direction was controlled in task and no-task conditions. When 'road sign' fixations were used (fixed position relative to the road) participants steered towards the direction of their gaze, a finding in line with the Active Gaze model of steering (Wilkie et al., 2008). When a cognitive load was added the bias towards the fixation point did not increase; higher bias towards the point of gaze would indicate that other sources of visual information (such as road-edges or retinal flow) are being weighted less (Kountouriotis et al., 2012; Wilkie \& Wann, 2003). It appears that the overall processing of the scene did not alter with the addition of cognitive load since participants were largely unaffected by the different cognitive task conditions in terms of steering bias as well as steering variability. However, when IVIS fixations were used (fixed position relative to the vehicle), participants had lower RMS steering error under the cognitive load conditions. This seems to indicate that the direction of gaze can interact with cognitive load and influence the resources available to steer. Our fixation points in Experiment 1 resembled IVIS displays, but similar properties can be seen in head-up displays (HUDs) in terms of the constant angle to the driver regardless of the trajectory they take. One crucial difference is that HUDs can display information in front of the driver rather than requiring them to turn their head towards an IVIS which is preferable since it does not take gaze away from the scene and potential hazards. One possible 
limitation of this method of displaying information is that during bend negotiation a HUD would direct gaze towards the direction of instantaneous heading rather than the drivers' future path. It is not safe to assume that such shifts in gaze are safe, and further experiments are needed in order to clarify any detriments/improvements in driving behaviour associated with HUDs.

It should be highlighted that it is currently unclear why RMS error and steering reversals decrease when a cognitive load is added to the steering task. Macdonald and Hoffmann (1980) attempted to explain the negative relationship between concurrent task difficulty and reversal rates in terms of participants removing attention from the steering task. If participants fail to attend the steering task then fewer corrective steering adjustments would be made (and so fewer reversals). In line with this, Jamson and Merat (2005) found that as the demands of a concurrent auditory task increased, lane variation (a measure similar to RMS error) decreased. It should be noted that lane variation actually increased dur ing their 'visual task', but this may have simply been caused by head and eye movements sampling information from the screen presenting the visual task (the display was placed in the car in front of the radio control panels). Medeiros-Ward et al. (2014) argue that the reduction in steering variability under conditions of cognitive load is due to positive effects on performance of highly-automated tasks that do not require (and in fact will be impaired by) attention.

One difficulty with interpreting a low reversal rate is that this measure merely reflects few steering adjustments. In some cases this could be because of a lack of response to steering errors, but it could also be because there is simply no need to make a steering adjustment (i.e. steering performance is not errorful in the first place). To try to address this in our experiment reversals were defined as peaks in the opposite direction of the required steering response; in the literature, however, reversals can be defined as peaks in any direction ${ }^{3}$. Without comparing trajectories with the optimal steering line it can be difficult to draw firm conclusions from steering reversals. In the present experiments, participants were instructed to

\footnotetext{
${ }^{3}$ When reversals are defined in this way, it is very hard to distinguish between genuine reversals which adjust the steering course and variability in the steering response or, indeed, from simply unwanted noise.
}

stay in the centre of the road in order to be able to extract a useful measure of steering error and bias. Steering bias shows that trajectories were influenced by the point of gaze. We also found that the steering system is sufficiently flexible that participants can learn to steer away from the direction of fixation in order to bring visual information about the road towards central vision.

In Experiment 1, the effect of the two tasks on steering performance was similar. These results are in contrast to those of other studies (e.g., Engström et al., 2005; Jamson \& Merat, 2005) where steering performance was differentially affected by visual and cognitive load. One explanation for this contradiction may be the differences in gaze patterns across experiments. In previous work the visual tasks required gaze to be directed to a separate display and away from the road. In contrast the present experiments controlled gaze direction and we observed no differences in steering performance between different task conditions. Future studies in the area should take into account that differences in eye-movements between cognitive and visual task conditions might mediate any differences observed in steering performance.

In Experiment 2 offset fixations systematically biased steering, but the biases were not mediated by cognitive load; this finding suggests that there wasn't narrowing of the UFoV. The fixations used in Experiment 2 biased participants towards the direction of their gaze, which in turn reduced the angle of gaze. When the fixations were set at a constant eccentricity relative to the driver (Experiment 1), the angle could not be reduced. As such, it could be argued that the steering task in Experiment 1 was generally more challenging than in Experiment 2 and this could account for differences in the effect of cognitive tasks on steering. The differences in the fixation point properties map directly to real-world examples: information can be presented inside vehicles (for example in in-vehicle displays), in which case the driver cannot close down gaze angle, or outside of vehicles, for example road-signs, where gaze angle is not constant. Our data suggest that information presented on road signs may interfere less with steering, particularly if positioned in the direction you wish to travel. There are additional good reasons why looking away from your future path while driving for more than a few seconds poses increased risks 
in driver safety. For example, Liang, Lee, and Horrey (2014) in a large naturalistic driving study found that drivers looked away more from their future path in the seconds preceding a crash or a near miss. They argued that looking away from your future path for more than 1.7 seconds poses a risk hazard (Liang et al., 2014).

In conclusion, we have shown that gaze direction is a critically important factor in successful steering control. Concurrent cognitive tasks can have an effect on steering around bends, but this is dependent on the demands placed upon gaze and steering. We found no differences in steering performance across different cognitive tasks, presumably because we controlled for the direction of participants' gaze. Additionally, we have shown that gaze direction towards a point similar to an in-vehicle information system makes drivers more susceptible to cognitive interference as measured by reduced RMS error rates.

\section{Acknowledgments}

GK was funded by an EPSRC Doctoral Training Grant and subsequently by EPSRC EP/J002933/1 as a Research Fellow. The EPSRC had no involvement in the design, data collection, analysis and interpretation, in the writing of the report or in the decision to submit for publication. Thanks to John Wann for the loan of the eye-tracking equipment used in these experiments.

\section{References}

Ball, K. K., Beard, B. L., Roenker, D. L., Miller, R. L., \& Griggs, D. S. (1988). Age and visual search: Expanding the useful field of view. Journal of Optical Society of America, 5(12), 2210-2219.

Beilock, S. L., Carr, T. H., MacMahon, C., \& Starkes, J. L. (2002). When paying attention becomes counterproductive: Impact of divided versus skill-focused attention on novice and experienced performance of sensorimotor skills. Journal of Experimental Psychology: Applied, 8, 6-16.

Cooper, J., Medeiros-Ward, N., \& Strayer, D. L. (2013). The impact of eye movements and cognitive workload on lateral position variability in driving. Human Factors, 55, 1001-1014.
Drews, F. A., Pasupathi, M., \& Strayer, D. L. (2008). Passenger and cell-phone conversations in simulated driving. Journal of Experimental Psychology: Applied, 14, 392-400.

Engström, J., Johansson, E., \& Östlund, J. (2005). Effects of visual and cognitive load in real and simulated motorway driving. Transportation Research Part F: Traffic Psychology and Behaviour, 8, 97-120.

Harley, C., Wilkie, R. M., \& Wann, J. P. (2009). Stepping over obstacles: attention demands and aging. Gait Posture, 29, 428-432.

Jamson, A. H., \& Merat, N. (2005). Surrogate in-vehicle information systems and driver behaviour: Effects of visual and cognitive load in simulated rural driving. Transportation Research Part F: Traffic Psychology and Behaviour, 8, 79-96.

Kountouriotis, G. K., Floyd, R. C., Gardner, P. H., Merat, N., \& Wilkie, R. M. (2012). The role of gaze and road edge information during highspeed locomotion. Journal of Experimental Psychology: Human Perception and Performance, 38(3), 687-702.

Kubose, T. T., Bock, K., Dell, G. S., Garnsey, S. M., Kramer, A. F., \& Mayhugh, J. (2006). The effects of speech production and speech comprehension on simulated driving performance. Applied Cognitive Psychology, 20, 43-63.

Land, M. F., \& Horwood, J. (1995). Which parts of the road guide steering? Nature, 377(6547), 339-340.

Land, M. F., \& Lee, D. N. (1994). Where we look when we steer. Nature, 369, 742-744.

Liang, Y., Lee, J., \& Horrey, W. (2014). A looming crisis: The distribution of off-road glance duration in moments leading up to crashes/nearcrashes in naturalistic driving. Proceedings of the Human Factors and Ergonomics Society 58th Annual Meeting, 58, 2102-2106.

Macdonald, . W. A., \& Hoffmann, E. R. (1980). Review of relationships between steering wheel reversals rate and driving task demand. $\mathrm{Hu}$ man Factors, 22(6), 733-739.

McLean, J. R., \& Hoffmann, E. R. (1975). Steering reversals as a measure of driver performance and steering task difficulty. Human Factors, 17, 248-256. 
Medeiros-Ward, N., Cooper, J. M., \& Strayer, D. L. (2014). Hierarchical control and driving. Journal of Experimental Psychology: General, 143, 953-958.

Merat, N., Anttila, V., \& Luoma, J. (2005). Comparing the driving performance of average and older drivers: The effect of surrogate in-vehicle information systems. Transportation Research Part F: Traffic Psychology and Behaviour, 8, 147166.

Merat, N., \& Jamson, A. H. (2008). The effects of stimulus modality on signal detection: Implications for assessing the safety of in-vehicle technology. Human Factors, 50(1), 145-158.

Norman, D. A., \& Shallice, T. (2000). Attention to action: Willed and automatic control of behavior. In M. S. Gazzaniga (Ed.), Cognitive Neuroscience: A reader. Oxford: Blackwell Publishers Ltd.

Raw, R. K., Kountouriotis, G. K., Mon-Williams, M., \& Wilkie, R. M. (2012). Movement control in older adults: does old age mean middle of the road? Journal of Experimental Psychology: Human Perception and Performance, 38(3), 73545.

Readinger, W. O., Chatziastros, A., Cunningham, D. W., Bülthoff, H. H., \& Cutting, J. E. (2002). Gaze-eccentricity effects on road position and steering. Journal of Experimental Psychology: Applied, 8(4), 247-258.

Recarte, M. A., \& Nunes, L. M. (2003). Mental workload while driving: effects on visual search, discrimination, and decision making. Journal of Experimental Psychology: Applied, 9, 119-137.

Reimer, B. (2009). Cognitive task complexity and the impact on drivers' visual tunneling. Transportation Research Record, 2138, 13-19.

Robertshaw, K. D., \& Wilkie, R. M. (2008). Does gaze influence steering around a bend? Journal of Vision, 8(4), 18.1-13.

Salvucci, D. D., \& Beltowska, J. (2008). Effects of memory rehearsal on driver performance: Experiment and theoretical account. Human Factors, 50(5), 834-844.

Salvucci, D. D., \& Gray, R. (2004). A two-point visual control model of steering. Perception, 33(10), 1233-1248.
Shiffrin, R. M., \& Schneider, W. (1977). Controlled and automatic human information processing: II. Perceptual learning, automatic attending, and a general theory. Psychological Review, 84, 127-190.

Strayer, D. L., \& Drews, F. A. (2004). Profiles in driver distraction: Effects of cell phone conversations on younger and older drivers. Human Factors, 46, 640-649.

Strayer, D. L., Drews, F. A., \& Johnston, W. A. (2003). Cell phone-induced failures of visual attention during simulated driving. Journal of Experimental Psychology: Applied, 9, 23-32.

Tapp, K. M., \& Logan, G. D. (2011). Attention to the hands disrupts skilled typewriting: The role of vision in producing the disruption. Attention, Perception, \& Psychophysics, 73, 2379-2383.

Victor, T. W., Engström, J., \& Harbluk, J. (2008). Distraction assessment methods based on visual behavior and event detection. In M. Regan, J. Lee, \& K. Young (Eds.), Driver distraction: Theory, effects and mitigation. CRC Press.

Victor, T. W., Harbluk, J. L., \& Engström, J. (2005). Sensitivity of eye-movement measures to invehicle task difficulty. Transportation Research Part F: Traffic Psychology and Behaviour, 8, 167190.

Wann, J. P., Swapp, D., \& Rushton, S. K. (2000). Heading perception and the allocation of attention. Vision Research, 40(18), 2533-2543.

Wilkie, R. M., Kountouriotis, G. K., Merat, N., \& Wann, J. P. (2010). Using vision to control locomotion: Looking where you want to go. Experimental Brain Research, 204(4), 539-547.

Wilkie, R. M., \& Wann, J. P. (2002). Driving as night falls: The contribution of retinal flow and visual direction to the control of steering. Current Biology, 12(23), 2014-2017.

Wilkie, R. M., \& Wann, J. P. (2003). Controlling steering and judging heading: Retinal flow, visual direction, and extraretinal information. Journal of Experimental Psychology: Human Perception and Performance, 29(2), 363-378.

Wilkie, R. M., \& Wann, J. P. (2005). The role of visual and nonvisual information in the control of locomotion. Journal of Experimental Psychology: Human Perception and Performance, 31(5), 901-911. 
Wilkie, R. M., Wann, J. P., \& Allison, R. S. (2008). Williams, L. J. (1985). Tunnel vision induced by a Active gaze, visual look-ahead, and locomotor control. Journal of Experimental Psychology: Human Perception and Performance, 34(5), 1150-1164.

Williams, L. J. (1982). Cognitive load and the functional field of view. Human Factors, 24(6), 683-692. foveal load manipulation. Human Factors, 27, 221-227.

Received December 19, 2014 Revision received May 07, 2015 Accepted July 16, 2015 